\title{
Threshold Bloodstain Recognition Method Based on Hyper-spectral Imaging Technology
}

\author{
Nannan $\mathrm{Hu}^{1}$, Yunuan Wang ${ }^{2}$, Xiaofei $\mathrm{Li}^{1}$, Yonglei Liu ${ }^{1}$, Jingjing Wang ${ }^{1}$, Liren Zhang ${ }^{1}$, Yuefeng Zhao ${ }^{1, *}$ \\ 1. College of Physics and Electronic Science, Shandong Normal University, Jinan, China \\ 2. China Jiaotong University, Beijing, China \\ *Corresponding author is Yuefeng Zhao, emil:zhaoyuefeng@126.com
}

\begin{abstract}
The identification of bloodstain is one of the most important approaches in obtaining evidence in criminalistics. A threshold method based on spectral co-efficient and interclass variance is proposed in this paper, it is a non-contact, non-destructive method for quickly identifying bloodstains. The spectra of bloodstains and other suspected substances were all extracted from their hyper-spectral image. Then calculate the correlation coefficients of these spectral and interclass variances, analyze the differences between substances. The best blood recognition threshold was determined as 0.9. After preprocessing for eliminating systematic errors, experiments with the threshold 0.9 are carried out to identify bloodstains on the calico and red T-shirt. The method can remarkably identify the bloodstain from other non-blood substances both quickly and efficiently. The blood extraction rate can reach to $93.35 \%$ and $89.19 \%$, respectively. It is an important step toward the implementation of bloodstain non-contact and non-destructive identification in forensic casework.
\end{abstract}

Keywords: Hyper-spectral imaging system, Spectral characteristics, Image processing, Threshold method, Bloodstains recognition. 
Forensic biochemistry analytical technologies permit a significant amount of genetic information to be obtained from a biological stain found at a crime scene. Bloodstains are the most common biological stain in a violence incident. Bloodstains present one of the most useful physical evidences at the scene of a violent crime. From the crime scene investigation point of view, the age of a bloodstain can provide important information about the nature of the crime to reconstruct the scene. Furthermore, DNA or RNA extracted from the bloodstains is also an evidence for the investigator to identify the suspects or victims in the violence incident. Hence, the age of a blood stain is able to accurately provide significant forensic information.

Currently, the conventional methods being applied to bloodstain identification include Kastle-Meyer $(\mathrm{KM})$, Leuco-malachite Green (LMG), and luminol tests [1,2]. The luminol test is first used for the identification of bloodstains in [3]. The test is very sensitive and the result is obvious when the blood is located on fabric and carpet with absorbent substrates. However, this approach has some inherent defects. For example, it may produce false positives, create problems for the subsequent tests of DNA [3-5], as well as lead to the dilution of the stain. In addition, the use of such chemical is hazardous to the health of the investigators. Likewise, the same weakness can be also found in the KM and LMG $[1,2]$. As for alternative approaches, high performance liquid chromatography (HPLC) [6, 7], Oxygen electrode method [8], electron paramagnetic resonance method [9], Atomic microscopy [10], and RNA degradation measurement are used for the identification of bloodstains $[11,12]$. Unfortunately, the above-mentioned methods have been used in practical investigations for a long time and their testing results are somehow inaccurate. The other weakness includes that these methods require a sample from primitive bloodstains to be collected for the experimental test, and such requirement could certainly damage the original bloodstain, which is valuable evidence 
for the criminal investigation. Furthermore, these methods usually involve more complex biochemical reactions, realty increasing the time needed to obtain results [6].

Since it is a new combination of molecular biology and physics, spectroscopic methodology is a non-destructive and non-contact bloodstain identification method that is gradually being used in forensic investigation. Many approaches based on the spectroscopic method have been proposed, such as the detection of latent fingerprints $[13,15]$, physical evidence, as well as the identification of the bloodstain using imaging techniques. Schuler investigates a non-destructive approach for the identification of bloodstains on black fabrics using near-infrared hyper-spectral imaging [9]. A hyper-spectral camera records the back-scattered light of many narrow wavelength bands and generates a data set of monochrome images for each wavelength. The numerical results demonstrate that the contrast between bloodstains and black fabrics is enhanced by the hyper-spectral images operating in the wavelength ranging from $650 \mathrm{~nm}$ to $1100 \mathrm{~nm}$ and from $960 \mathrm{~nm}$ to $1650 \mathrm{~nm}$ [16]. In [17], Edelman focuses on the visible reflectance of hyper-spectral imaging in the wavelength ranging from $400 \mathrm{~nm}$ to $720 \mathrm{~nm}$. This approach is able to visualize bloodstains on 12 different black fabrics. Li was successful in the discrimination of bloodstains from nine red substances that were dropped on the paper based on the Soret $\gamma$ band absorption in haemoglobin between $400 \mathrm{~nm}$ and $500 \mathrm{~nm}[18,19]$. It can be seen that approaches presented in [16-19] demonstrate that hyper-spectral imaging technology can be widely used in non-destructive testing of bloodstain in different wavelength ranges.

This study presents a novel approach for non-contact detection and identification of bloodstains using visible near infrared wavelength hyper-spectral imaging based on the reflectance of different substances. The research focuses on the impact of visible reflectance related to the hyper-spectral 
imaging in wavelength ranging from $400 \mathrm{~nm}-800 \mathrm{~nm}$ to the visualization of bloodstain, as well as other similar blood substances on two different fabrics. The significance of this research is that a threshold method based on spectral co-efficient and inter-class variance is proposed in this paper, which can be applied in crime scene for the identification and detection of bloodstain, including the suspected blood or even the incident scene being washed. It is an efficient, non-destructive technology for quickly identifying bloodstains. The bloodstain identification approach is based on a visible near infrared reflectance hyper-spectral imaging system operating in wavelength, combined with spectral co-efficient and inter-class variance calculate, which finally allows the best bloodstain recognition threshold to be determined as 0.9 . The proposed hyper-spectral image processing approach is evaluated by two sets of bloodstain samples on the calico and the red T-shirt, separately. A comparison of the numerical results using the proposed approach and the other wellknown conventional approaches is presented.

\section{Material and methods}

\section{Hyper-spectral imaging system}

The hyper-spectral imaging system (Gaia Sorter) used by this study consists of spectral camera (INFINITY-3), light sources for scene illumination, motorized precision translation stage, and a computer. The spectral camera is capable of operating in a wavelength range from $400 \mathrm{~nm}$ to 1000 $\mathrm{nm}$. This system is a $1392 \times 1040$ pixel complementary metal oxide semiconductor device that is able to detect elements of Cooled CCD with relative aperture of F/2.4 and the slit size of $30 \mu \mathrm{m}$ (W) x $8.98 \mathrm{~mm}$ (L). Also, four tungsten halogen light sources are able to provide incident light. In this study, the radiant output power provided by the light sources is able to cover a range of approximately $100 \mathrm{~cm}^{2}$; however, the light power within the wavelength ranging from $400 \mathrm{~nm}$ to $1000 \mathrm{~nm}$ is about $10 \mathrm{~W}$. Thus, it gives a power density of approximately $100 \mathrm{~mW} / \mathrm{cm}^{2}$. The hyper- 
spectral image is captured in the range of $400 \mathrm{~nm}$ and $1000 \mathrm{~nm}$ in wavelength with spectral subsampling at approximately $1.2 \mathrm{~nm}$ intervals in a format of 520 bands for an image cube.

Figure 1 shows the hypercube of a bloodstain on white fabric. Hyper-spectral imaging techniques are based on the acquisition of spectral data from each pixel of an image rather than a single point, forming a three-dimensional multivariate array of data with two spatial dimensions (x, y) and one wave length dimension $(\lambda)$ [20], which is also known as a hypercube. In comparison with general imaging technology, it is highlighted with multi-band, narrow spectral range, and a continuous band.

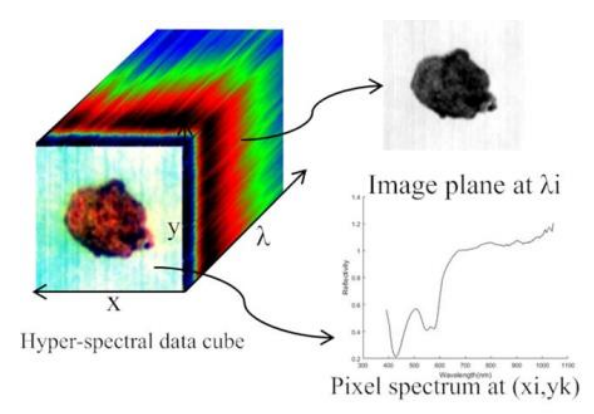

Fig.1. Hypercube of a bloodstain on white fabric, with two spatial $(x, y)$ and one wavelength $(\lambda)$ dimension. From the hypercube an image planes shown for one wavelength $(\lambda \mathrm{i})$ and a spectrum is obtained from one pixel (xi,yk ).

\section{The criteria for bloodstain identification}

1. The reflectance spectrum of bloodstain in the visible region is dominated by the spectrum of haemoglobin present in blood [21, 22]. It mainly consists of a strong narrow absorption center at approximately $415 \mathrm{~nm}$, referred to as the Soret band, with two weaker and broader absorptions between $500 \mathrm{~nm}$ and $600 \mathrm{~nm}$, which are known as the $\beta$ and $\alpha$ bands, respectively.

Figure 2 shows the reflectance spectrum of a fresh bloodstain. Other red substances absorb in the region of the visible spectrum between $400 \mathrm{~nm}$ and $500 \mathrm{~nm}$, the width of their absorption is typically much wider rather than a center at $410 \mathrm{~nm}$ [19]. Therefore, different types of substances have different reflectance spectrums in a specified wavelength range. This feature 
can be applied to identify and discriminate bloodstains from other substances having a very similar color.

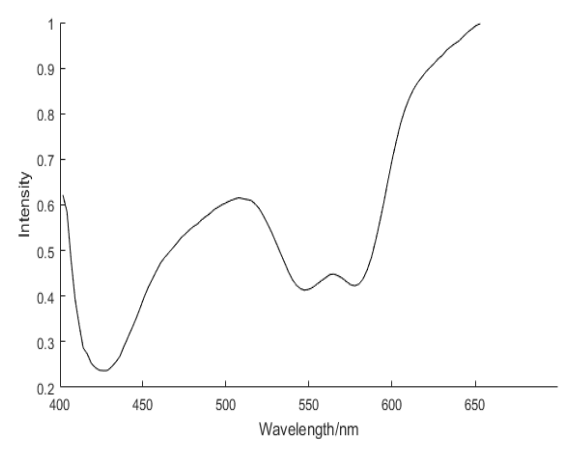

Fig.2.The reflectance spectrum of a fresh blood stain

2. The further investigation between bloodstain and other non-blood substances can be processed by a reflectance spectrum comparison of the suspected bloodstain and references, including both bloodstains and non-bloodstains using the statistical co-efficiency of determination, denoted as R2. The reference library of bloodstains includes aged bloodstains from 1 hour to 40 days. The reference library of non-bloodstains consists of approximately 45 reddish-color substances, which can likely be real blood. These non-bloodstains include coffee, red neutral pen, cola, chili oil, and soy sauce. The value of statistical co-efficiency R2 is calculated using the reflectance spectrum for all the bloodstains and the non-blood stains in the reference libraries. The calculation of R2 is shown as equation (1).

$$
R 2=\frac{E(X Y)-E(X) E(Y)}{\sqrt{E\left(X^{2}\right)-E^{2}(X)} \sqrt{E\left(Y^{2}\right)-E^{2}(Y)}}
$$

where $\mathrm{E}$ refers to mathematical expectation, $\mathrm{X}$ is the standard blood spectrum signal and $\mathrm{Y}$ represents the spectral signal of the stain to be identified. It has the same meaning as the Pearson function, describes the degree of linear correlation between the two variables.

The proportion of blood pixels in hyper-spectral images is recorded as $\omega_{1}$, and its average gray value is recorded as $\mu_{1}$. The proportion of other stain pixels is recorded as $\omega_{2}$, and its average gray value is recorded as $\mu_{2}$. 
The inter-class variance between bloodstain and other stains can be obtained from:

$$
G=\omega_{1} \times \omega_{2} \times\left(\mu_{1}-\mu_{2}\right)^{2}
$$

Combined considerations R2 and G, select the co-efficiency R2 that maximizes interclass variance between bloodstain and other stains as the decisive threshold for distinguishing bloodstain from other stains.

3. Threshold method is used to distinguish bloodstains from other stains. Pixels in the image of reflectance spectrum satisfying the conditions in criteria (1) and (2) are marked white, while other pixels are marked black. Therefore, the bloodstains can be easily identified in the reflectance spectrum image. Moreover, the blood extraction rate $r$ can be calculated by formula (3).

$$
r=\frac{N_{\text {extraction }}}{N_{\text {bl oodstain }}} \times 100 \%
$$

where $N_{\text {extraction }}$ the number of pixels is marked in white color, $N_{\text {bloodstain }}$ is the number of pixels of blood in the raw image data.

Experiment samples

The experiment is approved by School of Physics and Electronics, Shandong Normal University. All subjects gave their informed consent for inclusion before they participated in the study. The study was conducted in accordance with the Declaration of Helsinki, and the protocol was approved by the Ethics Committee and the Health Department.

Two sample sets are prepared in the experiment. The aim of this study is to explain the principle of using a hyper-spectral imaging system for bloodstain identification, as well as to demonstrate the significance that can be achieved by the system. The experiment includes two sample sets obtained from fabrics: calico sample set and red T-shirt sample set. The size of the sample is about $10 \mathrm{~cm}$ $\times 10 \mathrm{~cm}$. Their parameters are shown in Table1 and Table2, respectively. It should be noted that the substrate selected as the fabric sample is second-hand clothes, worn, and washed regularly prior to application of the blood. One drop of blood is deposited directly from the fingertip to each fabric sample, as well as other substances. Coffee, red ink, cola, chili oil, soy sauce, grape juice, and brown 
sugar water were selected as other substances for comparison because their colors are similar to bloodstains, making sure that there are bloodstains and other no-bloodstains for each sample set. All of the substances mentioned above were obtained from the supermarket and never opened before dropping stains.

Two group experiments were conducted in the hyper-spectral imaging laboratory.

1) Undiluted bloodstains, coffee, red ink, cola, chili oil, soy sauce, grape juice, and brown sugar water were deposited on white color fabric. The amount of these substances deposited on the substrate was controlled so these stains were visible.

2) A series of blind tests was conducted using the red T-shirt fabric with bloodstains and a mixture of other non-blood stains.

\section{Pre-processing of Hyper-spectral image}

Due to the system factors such as dark current, scattering, and so on, the hyper-spectral images directly obtained from the hyper-spectral system need a series of pre-processing before it can be applied to the recognition, statistics, or classification.

First, it is necessary to directly calibrate the image acquired by the Gaia Sorter because the hyperspectral imaging system generates a large amount of noise that is simultaneously acquired with the imaging signal. In this study, the black and white calibration was selected for the correction of the original image. The key mechanism in the black and white calibration was to calculate the calibration co-efficiency, that is:

$$
R=\frac{I_{S}-I_{D}}{I_{W}-I_{D}} \times 100 \%
$$


where $R$ refers to the relative spectrum reflectivity of the corrected hyper-spectral image, $I_{S}$ is the original hyper-spectral image obtained directly, $I_{D}$ indicates a black board calibration image with the reflectance of $0 \%$, and $\mathrm{I}_{\mathrm{W}}$ shows a white calibration image of the standard white board with a reflectance of $99 \%$. The performance of the black and white calibration can clearly be represented by the spectral curve, which shown in Fig.3.
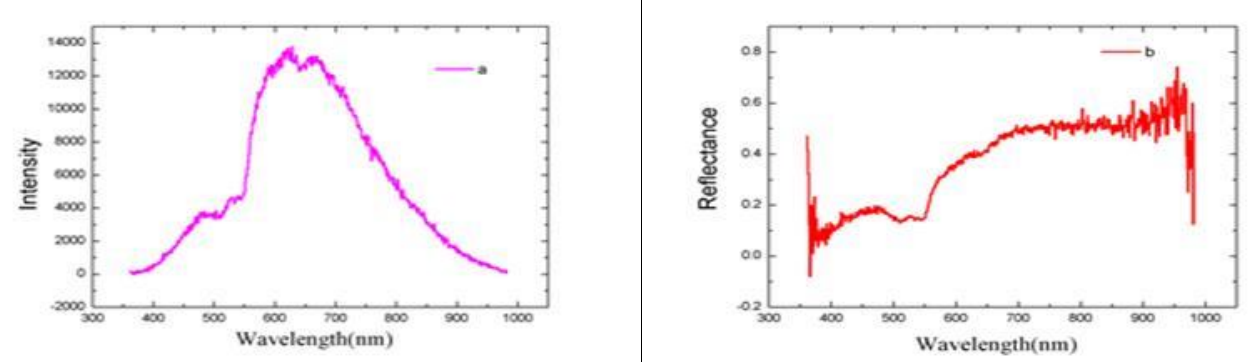

Fig.3. Curve a is reflectance spectral of the bloodstain before calibration, curve $\mathrm{b}$ is reflectance spectral after calibration.

Then, the Minimum Noise Fraction (MNF) was used to eliminate the noise produced in the hyperspectral imaging process. The key element is to determine the intrinsic dimension of the hyperspectral data, which can effectively filter the noise. It is clear that MNF is able to significantly reduce the noise and improve computing capacity required in the subsequent processing. The performance of MNF can clearly be represented by the spectral curve, as shown in Fig.4.
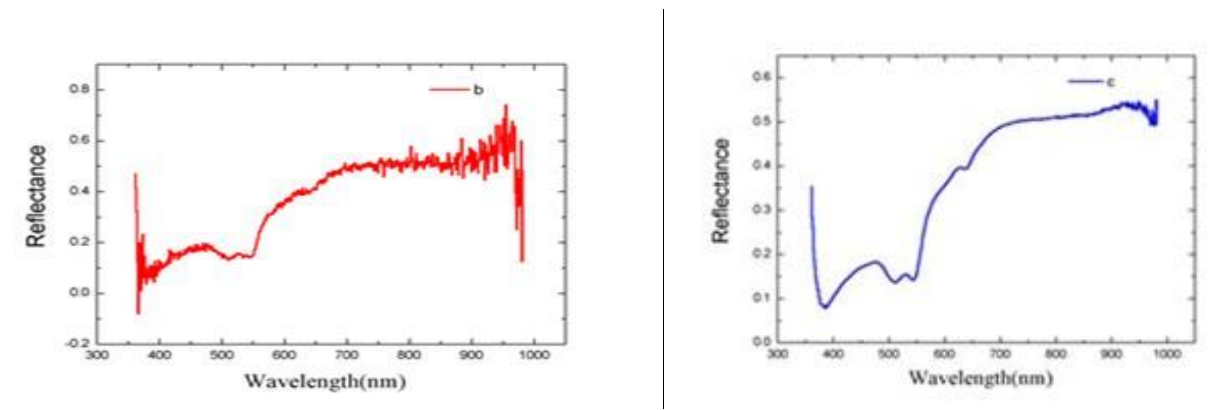

Fig.4. Curve $\mathrm{b}$ is reflectance spectral of the bloodstain before MNF curve $\mathrm{c}$ is reflectance spectral of the bloodstain after MNF 
According to the requirement of data analysis in terms of speed and efficiency, the hyper-spectral image needs to be segmented by regions, so as to extract the useful data areas of interest and to make the following procedure more succinct, eliminating the adverse effects of unrelated background on blood identification and analysis. Fig.5 shows the image after the selection of ROI. Bloodstain and blood analog stains are all selected and the background is deducted.

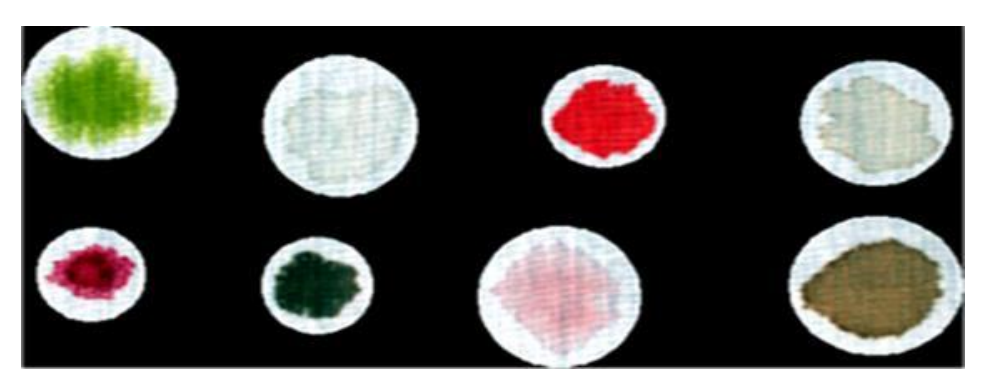

Fig.5. Extracted ROI of samples

On the one hand, the implementation of these pre-processing methods can eliminate a series of systematic errors caused by filters and dark currents. On the other hand, it is the first filtering of effective data, which reduces the size of data and makes subsequent analysis faster and more efficient.

\section{Results and Analysis}

\section{The experimental testing of bloodstains on the calico}

Figure 6 shows the mean spectral characteristics of bloodstain and other substances. It can be seen that the mean spectral curve of bloodstain has a strong narrow absorption center at approximately $415 \mathrm{~nm}$, which is referred to as the Soret band, and two distinct peaks between $500 \mathrm{~nm}$ to $550 \mathrm{~nm}$ in wavelength. The Soret band has a shift to shorter wavelength severely depending on time, which is called the blue shift phenomenon [23]. It can be seen that the bloodstain has its unique spectral value and unique trend of spectral change compared to the other substances, especially in the Soret 
band and bands from $500 \mathrm{~nm}$ to $550 \mathrm{~nm}$. Considering the blue shift in the Soret band, we take bands from $500 \mathrm{~nm}$ to $550 \mathrm{~nm}$ for deeper analyses.

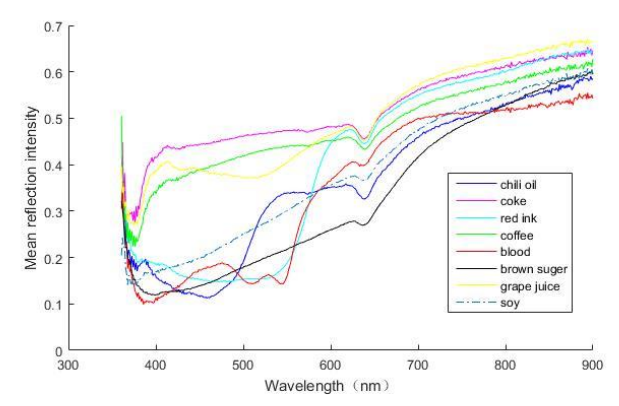

Fig.6 Mean reflection intensity of samples.

Based on the spectral characteristics of bloodstain - especially these distinct peaks between $500 \mathrm{~nm}$ to $550 \mathrm{~nm}$ in wavelength and criteria (2) testing on samples for an investigation of the optimum value of R2. When the test sample is a bloodstain, its reflectance spectrum is expected to be similar to the reflectance spectrum of bloodstains shown in the reference library. This means that the maximum value of $\mathrm{R} 2$ is expected to be close to 1 , indicating the better degree of association between standard bloodstain curves and pending recognition curve. Fig 7 shows the max and min value of R2. X-axis labeling represents different stains and bloodstains.
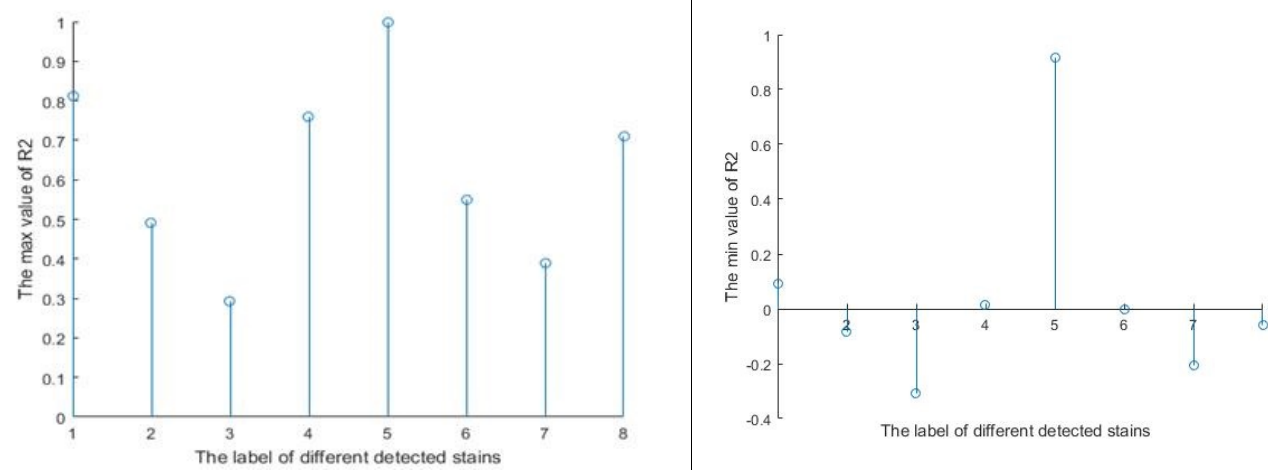

Fig.7 The max and min value of coefficient R2 between stains to be detected and standard value. 


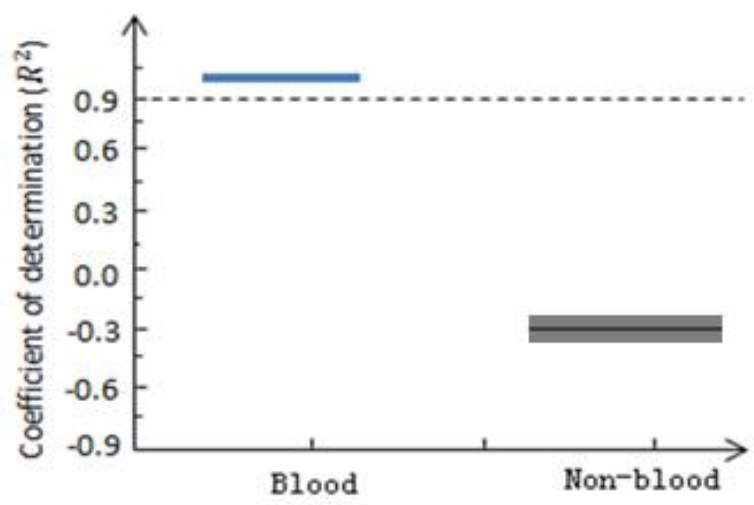

Fig.8. a box plot shows the coefficient of determination (R2) for reference blood stains and a library of non-blood stains. The line inside the box indicates the median value whilst the open square represents the mean value.

Figure 8 shows a box plot exhibiting the co-efficient of determination R2 for bloodstain and other non-blood substances. It is obvious that the co-efficient of determination R2 for bloodstain is close to 1 . By contrast, the co-efficient of determination R2 for other non- blood substances is far less than 1.

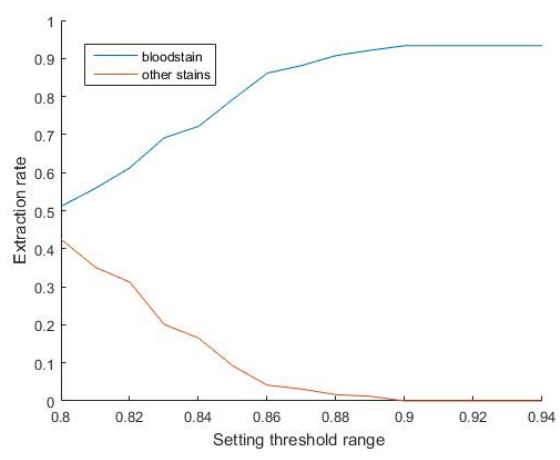

Fig.9. When selects different threshold, the extraction rate of bloodstain and other stains.

We selected a threshold from 0.80 to 0.94 based on spectral coefficient. Figure 9 respectively shows that the extraction rate of bloodstain and other stains in this threshold. When the threshold is less than 0.9 , other stains would interfere with the identification and extraction of bloodstain. Starting from 0.9 , the extraction rate of other stains become 0 , and the extraction rate of bloodstain up to the maximum value. Based on this phenomenon, Figure 8 is demonstrates that the bloodstain has been 
successfully identified by a threshold co-efficient of determination $\mathrm{R} 2=0.9$. Based on formula (3) in criteria (3), the blood extraction rate is about $93.35 \%$.

\section{The experimental testing of bloodstains on red color T-shirt}

A red color T-shirt is selected as the fabric for bloodstain identification experiments; the hyperspectral image after MNF and ROI processing is shown in Fig.10. The reason for selecting the red T-shirt as the fabric is that the bloodstain on a red color T-shirt may be hardly noticed by the naked eye. Therefore, such experimental test aims to evaluate the accuracy and efficiency of the proposed approach. In the experiment, the red T-shirt fabric contains eight stains, including chili oil, coke, red ink, coffee, bloodstain, sugar water, grape juice, and soy sauce. The experiment is conducted on blind testing basis, signifying that the nature of all stains on the red color T-shirt is independently blind to the experimental team. Considering spectral correlation differences, we can obtain the single band image shown in Fig 11. The stains correspond to the stains in Fig 10. The bloodstains were clearer than other stains, the same threshold value 0.9 is also applied to the treatment of the red T-shirt, and the recognition result is presented in Fig. 12.

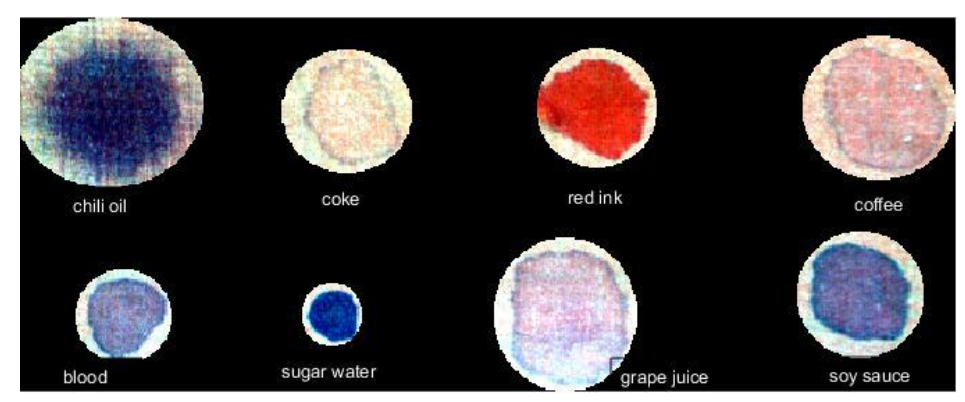

Fig.10. the hyper-spectral image of stains on red T-shirt after preprocessing and ROI extraction. 


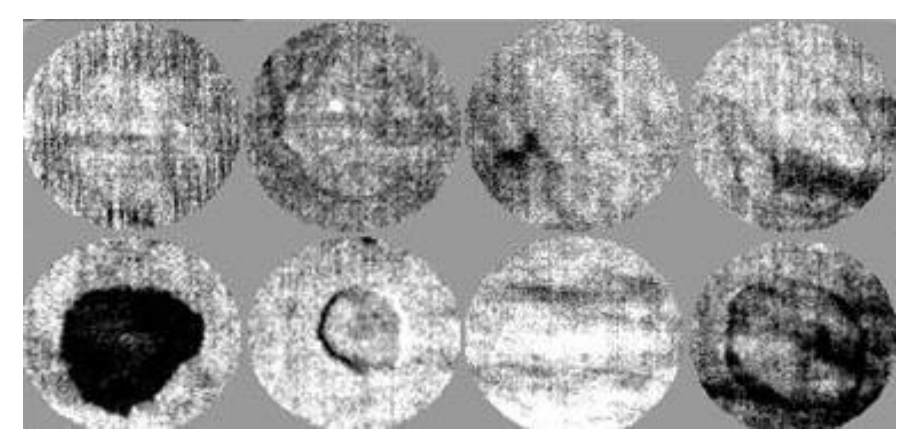

Fig.11 A single band image selected by spectral correlation.

Figure 12 shows the most bloodstain pixels of the reflectance spectral image satisfying the condition of R2>0.9, and they are all marked in white color by the proposed method. The rest of the pixels that do not contain blood composition have a co-efficient far less than 1 and are marked in black by a threshold 0.9. The bloodstain extraction rate is also calculated as the testing on calico, and in the red T-shirt bloodstain extraction rate can be up to $89.19 \%$.

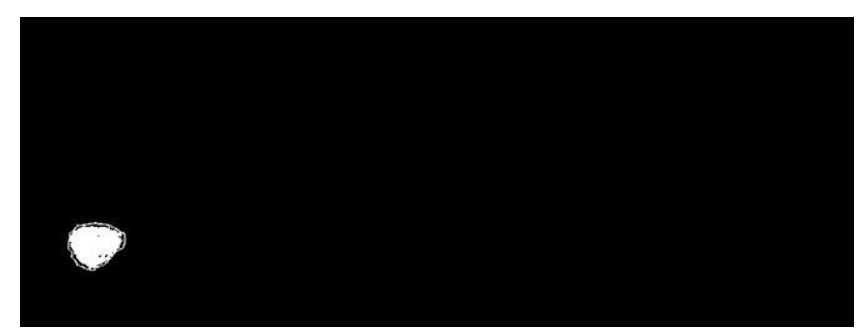

Fig.12.The result of bloodstain recognition.

\section{Conclusion}

Threshold based on spectral co-efficient and inter-class variance are proposed in this paper. It is an efficient non-destructive technology for quickly identifying bloodstains. The bloodstain identification approach is based on a visible near infrared reflectance hyper-spectral imaging system operating in wavelengths ranging from $400 \mathrm{~nm}$ to $800 \mathrm{~nm}$, combined with spectral characteristic coefficient and inter-class variance calculation. After a number of experimental tests, the extraction efficiency of bloodstains is compared for different thresholds. Finally, the optimal bloodstain recognition threshold is 0.9 . In order to eliminate some systematic errors, a series of pre-processing 
of directly obtained hyper-spectral images are carried out. Then, a series of experimental investigations are conducted at a threshold of 0.9 to identify bloodstains on calico and red T-shirt under blind testing condition. It can be seen that the proposed method is able to remarkably and efficiently identify the bloodstain from other non-blood substances. The blood extraction rate can reach up to $93.35 \%$ and $89.19 \%$, respectively. The numerical results obtained from experiments on blind testing basis are able to demonstrate the capability of using reflectance hyper-spectral imaging system in wavelength to visualize bloodstains on cotton cloth fabric for bloodstain identification purposes. These results illustrate the flexibility, availability, and convenience of the proposed recognition method.

\section{Funding}

National Natural Science foundation of China under Grant （No.81400285）; The key research and development foundation of Shandong Province under Grant

( No.2016GGX101016) ;Graduate Research Innovation Foundation of Shandong normal University in 2018, under Grant SCX201838.

\section{Author Contributions}

Data curation, Nannan Hu; Funding acquisition, Yuefeng Zhao; Investigation, Yunuan Wang and Xiaofei Li; Methodology, Jingjing Wang and Liren Zhang; Software, Yonglei Liu; Writing review \& editing, Nannan Hu.

\section{References}

1. Barni F, Lewis S. W , Berti A , Miskelly G. M , Lago G. Forensic application of the luminol reaction as a presumptive test for latent blood detection. TALANTA, 2007; 72(3): 896-913.

2. Tobe S. S, Watson N, Niamh Nic Daéid. Evaluation of six presumptive tests for blood, their specificity, sensitivity, and effect on high molecular-weight dna, J FORENSIC SCI, 2007; 52(1): 102-109.

3. Lytle L. T, Hedgecock D. G. Chemiluminescence in the visualization of forensic bloodstains, J FORENSIC SCI, 1978; 23(3): 550-562. 
4. Nesterenko P N, Jones P . Single-column Method of Chelation Ion Chromatography for the Analysis of Trace Metals in Complex Samples[J]. J CHROMATOGR A, 1997, 770(1):129-135.

5. Laux D. L. Effects of luminol on the subsequent analysis of bloodstains, J FORENSIC SCI, 1991, 36(5): 1512-1520.

6. Inoue H, Takabe F, Iwasa M, Maeno Y, Seko Y. A new marker for estimation of bloodstain age by high performance liquid chromatography, Forensic Sci Int,1992; 57(1): 17-27.

7. Andrasko J. The estimation of age of bloodstains by hplc analysis, J FORENSIC SCI,1997; 42(4): 601-607.

8. Matsuoka T, Taguchi T, Okuda J. Estimation of bloodstain age by rapid determinations of oxyhemoglobin by use of oxygen electrode and total hemoglobin, BIOL PHARM BULL,1995; 18(8): 1031-1035.

9. Fujita Y, Tsuchiya K, Abe S, Takiguchi Y, Kubo S. I. , Sakurai H. Estimation of the age of human bloodstains by electron paramagnetic resonance spectroscopy: long-term controlled experiment on the effects of environmental factors, Forensic Sci Int,2005; 152(1): 39-43.

10. Wu Y, Hu Y, Cai J, Ma S, Wang X, Chen Y. Time-dependent surface adhesive force and morphology of rbc measured by afm. MICRON, 2009; 40(3): 359-364.

11. Alrowaithi M. A Mccallum, N A, Watson N. D. A method for determining the age of a bloodstain, Forensic Sci Int, 2014; 23(4): 30-31.

12. Li B, Beveridge P, O'Hare W. T, Islam M. The estimation of the age of a blood stain using reflectance spectroscopy with a microspectro-photometer, spectral pre-processing and linear discriminant analysis, Forensic Sci Int, 2011; 212(1-3): 198-204. 
13. Camilla Ricci A. Steve Bleay, and Sergei G. Kazarian, Spectroscopic Imaging of Latent Fingermarks Collected with the Aid of a Gelatin Tape, ANAL CHEM, 2007. 79(15): 5771-5776.

14. Cadd S, Li B, Beveridge P, William T. O’Hare, Campbell A, Islam, M. The non-contact detection and identification of blood stained fingerprints using visible wavelength reflectance hyperspectral imaging: part 1, SCI JUSTICE, 2016; 56(3): 181-190.

15. Ricci C. and S.G. Kazarian, Collection and detection of latent fingermarks contaminated with cosmetics on nonporous and porous surfaces, SURF INTERFACE ANAL, 2010. 42(5): 386-392.

16. Edelman G., Manti V., Ruth S. M. V., Leeuwen T. V., Aalders M. Identification and age estimation of blood stains on colored backgrounds by near infrared spectroscopy, Forensic Sci Int, 2012; 220(1-3): 239-244.

17. Edelman G. J, Leeuwen T. G. V, Aalders M. C, Visualization of latent blood stains using visible reflectance hyperspectral imaging and chemometrics ， J FORENSIC SCI, 2015; 60(5):188-192. 18. Zhao Y, Hu N, Wang Y, Liu Y, Li X, Wang J. The application of near-infrared reflectance hyperspectral imaging for the detection and extraction of bloodstains, CLUSTER COMPUT,2018; 1(3):1-9.

19. Li B, Beveridge P, O'Hare W. T, Islam M. The application of visible wavelength reflectance hyperspectral imaging for the detection and identification of blood stains, SCI JUSTICE, 2014; 54(6): 432-438.

20. Sun H, Dong Y, Zhang P, Meng Y, Wen W, Li N, Accurate age estimation of bloodstains based on visible reflectance spectroscopy and chemometrics methods, IEEE PHOTONICS J, 2017; 9(1):1-15. 
21. Schuler R. L, Kish P. E, Plese C. A, Preliminary observations on the ability of hyperspectral imaging to provide detection and visualization of bloodstain patterns on black fabrics, $\mathrm{J}$ FORENSIC SCI, 2012; 57(6), 1562-1569.

22. Li B, Beveridge P, O'Hare W. T, Islam M. The age estimation of blood stains up to 30 days old using visible wavelength hyperspectral image analysis and linear discriminant analysis, SCI JUSTICE, 2013; 53(3), 270-277.

23. Hanson E K, Ballantyne J. A blue spectral shift of the hemoglobin soret band correlates with the age (time since deposition) of dried blood stains [J], PLOS ONE, 2010, 5(9):128-130. 
Table.1. List of parameters of the calico

\begin{tabular}{ll}
\hline Parameter name & Parameter values \\
\hline Material quality & Pure cotton \\
Component content & $100 \%$ \\
Technology & Printing and dyeing \\
Color & Primary colors/ natural color \\
Density & Increased density \\
Shrinkage rate & Less than 5\% \\
Grain style & Plain \\
Elastic force & Nonelastic \\
Thickness index & moderate \\
\hline
\end{tabular}

Table.2. List of parameters of the red T-shirt

\begin{tabular}{ll}
\hline Parameter name & Parameter values \\
\hline Material quality & cotton \\
Component content & more than $96 \%$ \\
Technology & printing and dyeing \\
color & red \\
density & increased density \\
shrinkage rate & less than $5 \%$ \\
tactile index & moderate \\
smoothness index & moderate \\
\hline
\end{tabular}

